\title{
Direct currents in power transformers
}

\author{
Milan Stork ${ }^{*}$, Daniel Mayer ${ }^{* *}$
}

\begin{abstract}
The article is devoted to the physical nature of geomagnetism, magnetic storms and methods of predicting their origin and deals with geomagnetic induced currents called GIC (Geomagnetically Induced Currents) and their effect on power transformers. A simplified, single-phase transmission system is described mathematically and its analysis was performed. Also the phenomenon of periodic semi-saturation of the magnetic circuit of the transformers resulting in current overload of the transformer windings, which can lead to thermal damage of the windings is explained. In addition, there is a significant deformation of the currents in the electrical system. The numerical solution of the system was verified by measurement on an electrical model.
\end{abstract}

Keywords: geomagnetic field, coronal mass ejection, geomagnetically induced current, non-linear transformer, magnetic semi-saturation

\section{Introduction}

Solar activity is manifested, among other things, by eruptions that lead to the release of coronary matter into an interplanetary space - this phenomenon is called CMA (Coronal Mass Ejection). If the particle flow CMA - called solar wind - approaches to the Earth, it will cause changes (variation) of the magnetic field at the Earth's surface. These phenomena are common, they are usually insignificant and do not affect life on Earth, nor the function of technical devices. However, strong solar flares can cause strong and rapid variations in the geomagnetic field, so called magnetic storms, which can negatively affect the function of technical systems (see $e g[1,2]$ ).

The more sophisticated technology a person uses, the more vulnerable this technique is, and the danger of strong magnetic storms is becoming more current. There are also pessimistic forecasts of the possibility of the emergence of extremely strong magnetic storms that could threaten contemporary civilization. The largest observed solar eruption, with the resulting very strong magnetic storm was registered by British astronomer R. Carrington in 1859. Since the introduction of electricity into practice was at the very beginning, the magnetic storm disconnected the telegraph network in the entire northern hemisphere but did not cause more damage. At present, when all human civilization is dependent on electrical and electronics engineering, a similar storm would cause immense damage. Magnetic storms occur mainly in the Nordic countries. Over the last few decades, magnetic storms have caused a number of local collapses of electrical systems, especially in the Nordic countries. A rich bibliography (see, for example, [3-6]) refers to it, the authors of which are mainly the operators of the affected systems.
Magnetic storms can not be prevented, but their threat can be predicted with some degree of proximity. Magnetic storms can damage cable telecommunication networks and satellite communications systems, thus blocking data transfers, disrupting navigation systems and thus jeopardizing the safety of air transport, may also interfere with the operation of signaling and safety equipment for rail transport. Magnetic storms induce GIC currents that endanger the stability of power systems and cause power outages. In the extreme case, GIC could cause the collapse of the power grid and possibly global chaos, with catastrophic consequences.

Geomagnetic variations have a time-random course, varying in order in tens of seconds, and therefore, according to the law of electromagnetic induction, the time course of the GIC is random. Compared to industrial frequency, it is changing very slowly - we are talking about quasi-stationary DC currents. GICs are therefore limited in the system only by line resistances, while the effects of inductances and capacities are negligible.

\section{Effects of geomagnetically induced currents on power transformers winding}

\subsection{Specification of the problem solved}

\section{Description of the simplified approach}

- Based on the geomagnetic predictions obtained by processing the information from the satellites, we know the approximate time course of the expected magnetic storm, ie the time course of the magnetic induction $B(t)$ that acts on the electrification system. From this and from a topological arrangement of electrical systems we compute (see [2]) the induced voltage and then the GIC in the system conductors. We will consider this as DC currents.

* Department of Applied Electronics and Telecommunications/RICE, stork@kae.zcu.cz, ** Department of Theory of Electrical Engineering, mayer@kte.zcu.cz, University of West Bohemia, Plzen, Czech Republic 


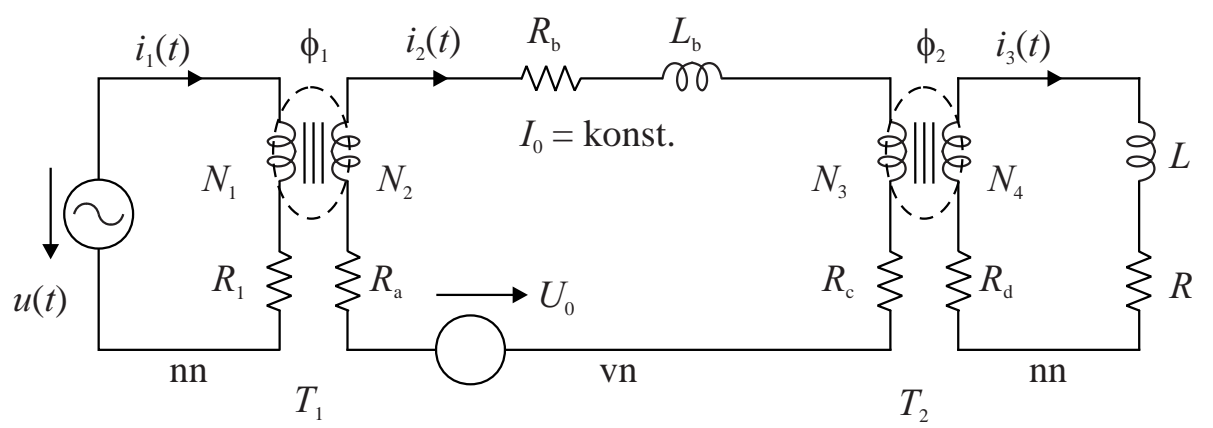

Fig. 1. Simplified transmission system

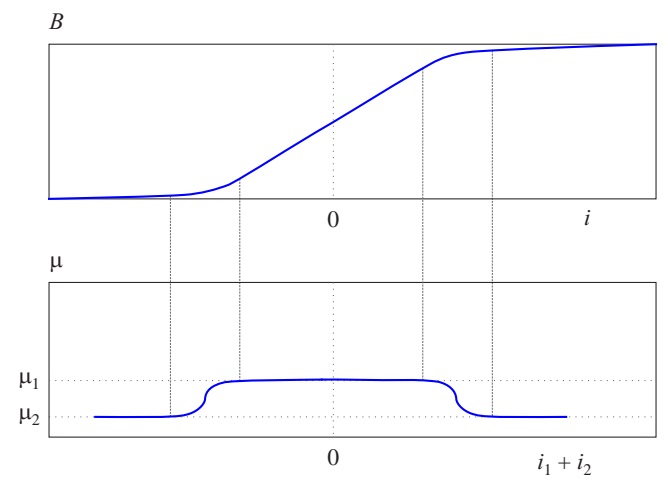

Fig. 2. The course of magnetization characteristics and permeability

- Replace the transmission system with the simple, single-phase model shown in Fig. 1. Generator, transformer, HV (high voltage) line, transformer, load. For both transformers we shall consider the non-linear magnetization characteristic $B=f(I)$, Fig. 2. From where we can determine the permeability of the magnetic circuit.

The other possibilities of modeling the transformer under magnetic storms are discussed in [6-10].

\subsection{Examining the properties of a simplified transmis- sion system}

\section{Mathematical model and its numerical solution}

In the circuit according to Fig. 1 the unknown quantities are currents: $i_{1}, i_{2}, i_{3}$ and magnetic fluxes $\Phi_{1}, \Phi_{2}$. We denote

$$
R_{2}=R_{a}+R_{b}+R_{c}, R_{3}=R_{d}+R
$$

Using Kirchhoff's laws, we find a mathematical model in the form of a system of nonlinear algebraic-differential equations, with zero initial conditions: $i_{1}(0)=i_{2}(0)=$ $i_{3}(0)=0$ and $\Phi 1(0)=\Phi 2(0)=0$.

$$
\begin{aligned}
& N_{1} \frac{\mathrm{d} \Phi_{1}}{\mathrm{~d} t}=-R_{1} i_{1}+u(t) \\
& N_{3} \frac{\mathrm{d} \Phi_{2}}{\mathrm{~d} t}=-R_{2} i_{2}-L_{b} \frac{\mathrm{d} i_{2}}{\mathrm{~d} t}-N_{2} \frac{\mathrm{d} \Phi_{1}}{\mathrm{~d} t}+u_{0}, \\
& L \frac{\mathrm{d} i_{3}}{\mathrm{~d} t}=-R_{3} i_{3}-N_{4} \frac{\mathrm{d} \Phi_{2}}{\mathrm{~d} t}, \\
& N_{1} i_{1}+N_{2} i_{2}-\frac{K_{1}}{\mu} \Phi_{1}=0 \\
& N_{3} i_{2}+N_{4} i_{3}-\frac{K_{2}}{\mu} \Phi_{2}=0
\end{aligned}
$$

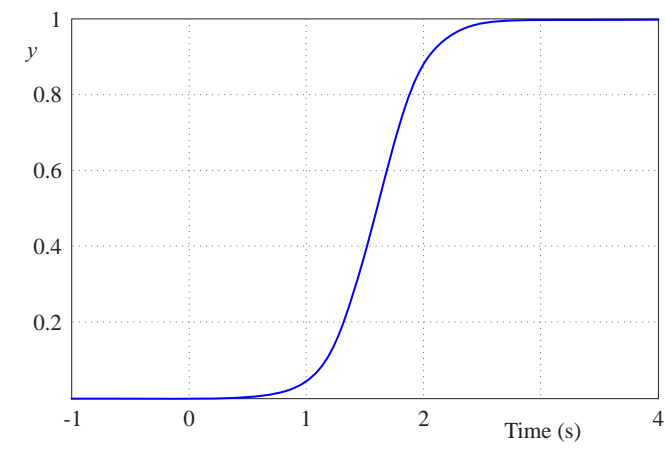

Fig. 3. Smooth function used instead of heaviside step function

Nonlinearities are only in the last two terms containing $\mu$-function and we choose $u(t)=U \sin (\omega t)$ as the power line excitation.

We are interested in a solution of steady state (eg after, several periods), when the transient phenomenon disappears. Since there were stability problems while solving the equations with a time-shifted Heaviside step function $U_{0} \mathbf{1}\left(t-t_{0}\right)$ we have used a smooth increase with "logsig" function instead. For $t_{0}=1 \mathrm{~s}: u_{0}(t)=U_{0}[1+\exp (5 t+$ $8)]^{-1}$, see Fig. 3.

The solution of this system was done by the MATLAB ode15i (Solve set of fully implicit differential equations) for the following inputs values: source $U=400 \mathrm{~V}, \omega=$ $2 \pi f, f=50 \mathrm{~Hz}$; transformers $T_{1}$ and $T_{2}$ are the equal, power $200 \mathrm{kVA}$, transformer voltage $6000 / 400 \mathrm{~V}, N_{1}=$ $N_{4}=40$ turns and $N_{2}=N_{3}=600$ turns, $R_{1}=0.40 \Omega$, $R_{2}=10.1 \Omega, R_{3}=1.1 \Omega, L_{l}=0.8 \mathrm{H}, L=2 \times 10^{-3}$ $\mathrm{H}, K_{1}=K_{2}=37$. Permeability dependence $\mu=f(I)$, Fig. 4, where $\mu_{1}=2.8 \times 10^{-3} \mathrm{H} / \mathrm{m}, \mu_{2}=0.3 \times 10^{-3}$ $\mathrm{H} / \mathrm{m}$. Nonlinear function $\mu=f(I)$ was approximated (providing the magnetizing current is in Ampers) by,

$$
\begin{gathered}
\mu(I)=3.22 \times 10^{-4}\left[1+8\left(1-\frac{1}{1+\exp \frac{I_{s}-I}{I_{k}}}\right.\right. \\
\left.\left.-\frac{1}{1+\exp \frac{I_{s}+I}{I_{k}}}\right)\right], \text { with } I_{s}=84, I_{k}=13
\end{gathered}
$$

Furthermore, their effective values, which are important for the evaluation of the influence of $u_{0}$ on the heating of transformer windings, were calculated, see Tab. 1. 


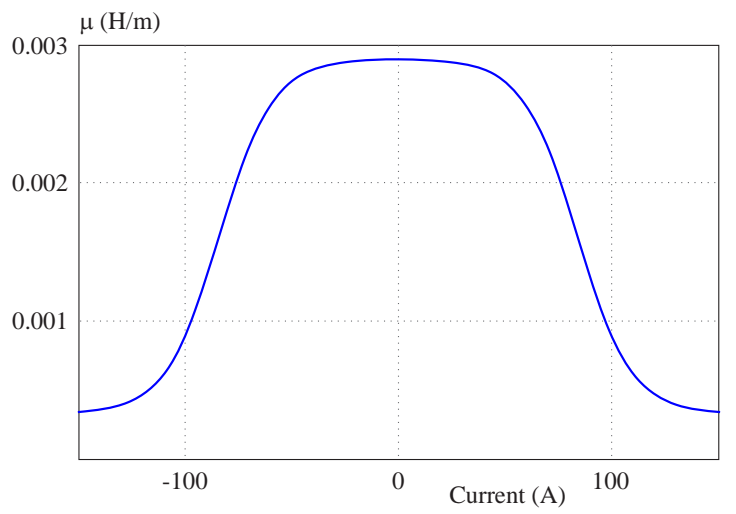

Fig. 4. The course of permeability: $\mu=f(I)$

The current $i_{1}$ is almost unchanged, depending on $U_{0}$ in steady state. On the other hand, the flow of the $i_{2}$ current changes significantly, its effective value is increased to $182 \%$. The $i_{3}$ current essentially does not change its effective value, but its shape is significantly deformed.

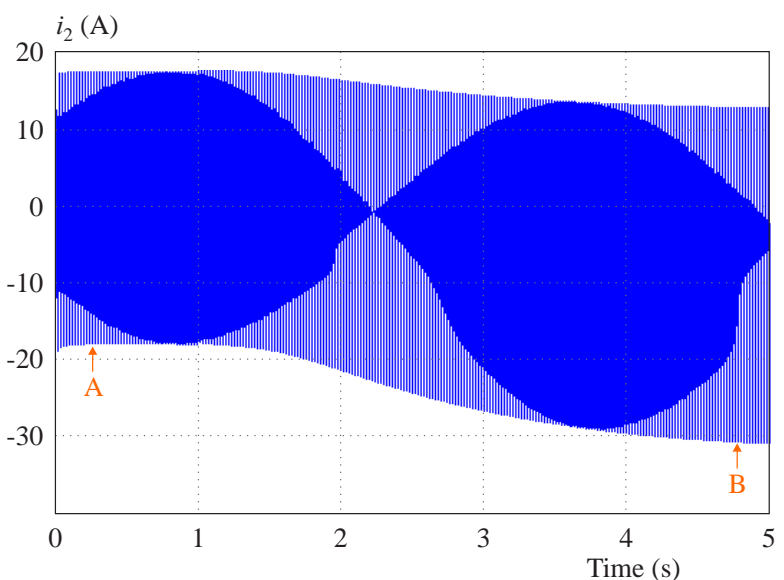

Fig. 5. Time evolution of current $I_{2}$ for $t \leq 1, U_{0}=0$ and for $t>1 U_{0}=100 \mathrm{~V}$. Voltage is increasing from 0 to $100 \mathrm{~V}$, according to (6), see Fig. 3. The zooms shown in Fig. 6 and Fig. 7 are taken approximately at time $\mathrm{A}$ and $\mathrm{B}$

\section{Experimental validation}

The circuit in Fig. 1 was modeled by the circuit in Fig. 8. This circuit illustrates three identical small single phase transformers $\operatorname{Tr}_{1}, \mathrm{Tr}_{2}, T_{\mathrm{C}} 230 \mathrm{~V}$ to $14 \mathrm{~V}, 0.2 \mathrm{~A}(2.8$ VA) used for experiment. Two transformers $T r_{1}$ and $T_{\mathrm{C}}$

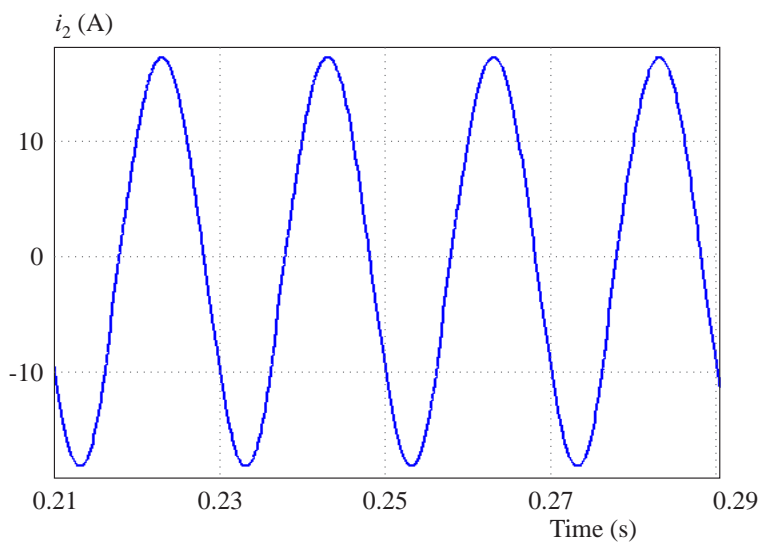

Fig. 6. Zoom of time evaluation of current $i_{2}$ for voltage $u_{0}=0$ (time is denoted as $\mathrm{A}$ in Fig. 5) were connected in such a manner that their secondary voltages oppose and as thus cancel. This is important for DC power supply connected to circuit. Instead of the induced voltage $u_{0}$, the GIC values, referred to as the current $I_{0}$, is given here. The time course of the currents $I_{1}, I_{2}, I_{3}$ was calculated from voltages $V_{\mathrm{A}}, V_{\mathrm{C}}$ and $V_{\mathrm{D}}$ on resistors $R_{1}, R_{3}$ and $R_{5}$. The results are shown in Fig. 9 and Fig. 10 and value of RMS currents in Table 2. The frequency spectrum of current $I_{3}$ for DC currents $I_{0}=0$ and for $I_{0}=0.5 \mathrm{~A}$ are shown in Fig. 11. Effective values of current $I_{2}$ is increased to $161 \%$ with DC current 0.5 A, and to $194 \%$ with $0.8 \mathrm{~A}$ (see Tab. 2).

Table 1. RMS currents without DC voltage and with DC voltage

\begin{tabular}{cccc}
\hline$u_{0}(\mathrm{~V})$ & $I_{1 \mathrm{RMS}}(\mathrm{A})$ & $I_{2 \mathrm{RMS}}(\mathrm{A})$ & $I_{3 \mathrm{RMS}}(\mathrm{A})$ \\
\hline 0 & 143 & 8.3 & 108 \\
100 & 166.5 & 15.1 & 127 \\
\hline
\end{tabular}

Table 2. RMS currents for zero DC current and currents 0.5 and $0.8 \mathrm{~A}$

\begin{tabular}{lccc}
\hline$I_{0}(\mathrm{~A})$ & $I_{1 \mathrm{RMS}}(A)$ & $I_{2 \mathrm{RMS}}(A)$ & $I_{3 \mathrm{RMS}}(A)$ \\
\hline 0 & 0.027 & 0.34 & 0.019 \\
0.5 & 0.084 & 0.55 & 0.022 \\
0.8 & 0.13 & 0.66 & 0.025 \\
\hline
\end{tabular}

\section{Discussion}

Numerical solution of the system (5) and the experimental verification of the circuit according to Fig. 8 both equally confirm the effect of the semi-saturation of transformers, if $u_{0} \neq 0$, or $I_{0} \neq 0$ there is current overload in $\mathrm{HV}$ lines and in winding of $\mathrm{HV}$ transformers.

The influence of magnetic storm on the power system is manifested by the induction of the GIC in the high-voltage lines. These currents are quasi-DC and cause the magnetic circuits of the transformers to be oversaturated. Since the HV windings have a large number of turns, even the small DC current can be enough to oversaturated the core. The magnetizing current then moves

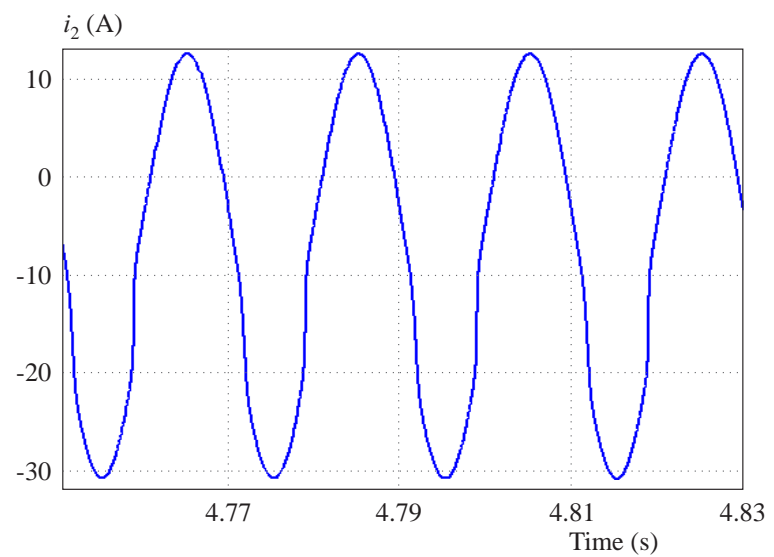

Fig. 7. Zoom of time evaluation of current $i_{2}$ for voltage $u_{0}=100$ $\mathrm{V}$ (time denoted as B in Fig. 5) 


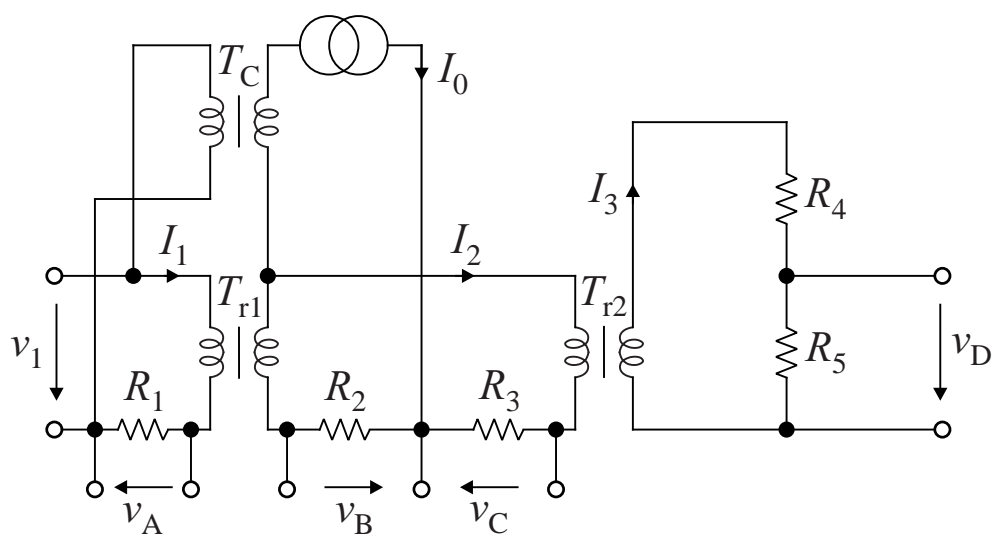

Fig. 8. The circuit diagram of experimental verification small transformers $T_{1}, T_{2}, T_{\mathrm{C}}: 230 \mathrm{~V}$ to $14 \mathrm{~V}, 0.2 \mathrm{~A}, R_{1}=100 \Omega$, $R_{2}=R_{3}=10 \Omega, R_{4}=2 k \Omega, R_{5}=180 \Omega, I_{0}$ controlled current source $0-1.5 \mathrm{~A}, V_{\mathrm{A}}, V_{\mathrm{B}}, V_{\mathrm{C}}, V_{\mathrm{D}}-$ voltages across resistors

in half-period in the unsaturated (linear) part of the magnetization characteristic, and in second half-period in supersaturated part. There is a semi-saturation of the magnetic circuit of the transformer. This phenomenon is manifested in the electricity system as follows:

- Transformers $T_{1}$ and $T_{2}$ have an overloaded HV winding (the effective value of currents is increased) and excessive warming occurs. However, GIC currents have a different character than short-circuit currents in the system whom we have rich experience, and with the appropriate protection we can face them. While shortcircuit currents are fast and threaten the winding not only thermally, but primarily by mechanical impact, the GIC acts in the order of minutes or even much longer. GIC measurements and their thermal effects on power transformers are shown, for example in [7].

- The quality of the transmitted electric energy due to the magnetic storm drops strongly. As an example, in Figs. 9, 10, the waveform $i_{3}$ is obtained by measuring the circuit shown in Fig. 8. The deterioration of the quality is evident from the frequency spectrum of the current $i_{3}(t)$, Fig. 11. Similar results, measured on actual transformers, are given, for example, in [8-11].

Other negative effects of magnetic storms: If the transformer is operating in semisaturation mode, its reactive

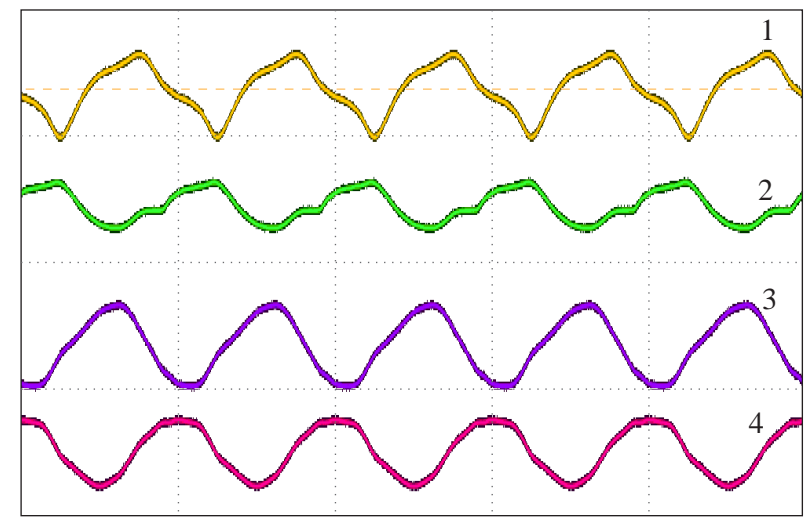

Fig. 9. Time-dependent voltages; from top to bottom: $V_{\mathrm{A}}, V_{\mathrm{B}}, V_{\mathrm{C}}$, $V_{\mathrm{D}}$ results of experimental solution according Fig. 8 for DC current $I_{0}=0.5 \mathrm{~A}$ power is increased. The rise of higher harmonics results in an increase in Joule losses in the winding, in the iron of the transformers and it the transformer tank. The presence of higher harmonics also increases the magnetic leakage of the transformer, thereby increasing the loss of eddy currents in the transformer tank, which increase its warming. Higher harmonics further increase the noise of the transformer, eg [7] indicates an increase in noise of up to $20 \mathrm{~dB}$.

\section{Conclusion}

An analysis of the semi-saturation phenomenon in transformer brings insights into designers of power transformers. The destructive effect of magnetic storms on the power system can be partly suppressed by the transformer design. By appropriately selecting the magnetic circuit of the transformer (core or shell, see, for example, [10]) and suitable connecting of the winding it is possible to achieve a more robust transformer that suppresses the occurrence of magnetic semisaturation however, but at the expense of higher production and operating costs. Another effective protection of transformers consists in obtaining a valid

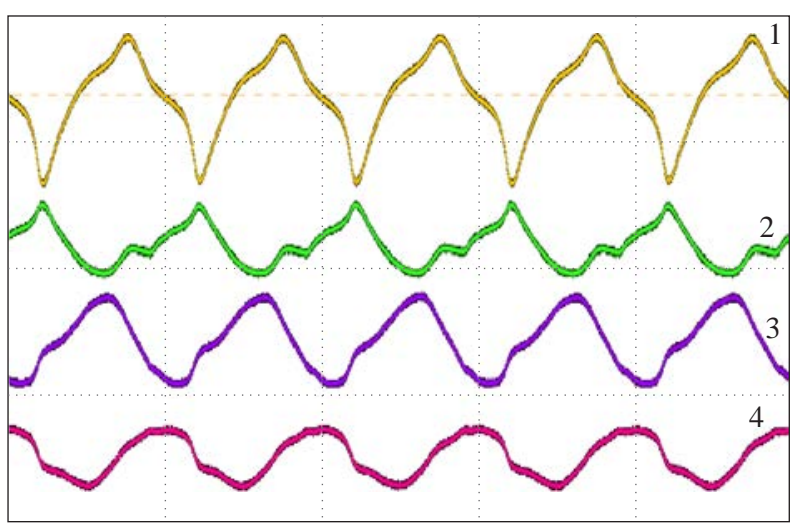

Fig. 10. Time-dependent voltages from top to bottom: $V_{\mathrm{A}}, V_{\mathrm{B}}, V_{\mathrm{C}}$, $V_{\mathrm{D}}$ results of experimental solution according Fig.8 for DC current $I_{0}=0.8 \mathrm{~A}$ 


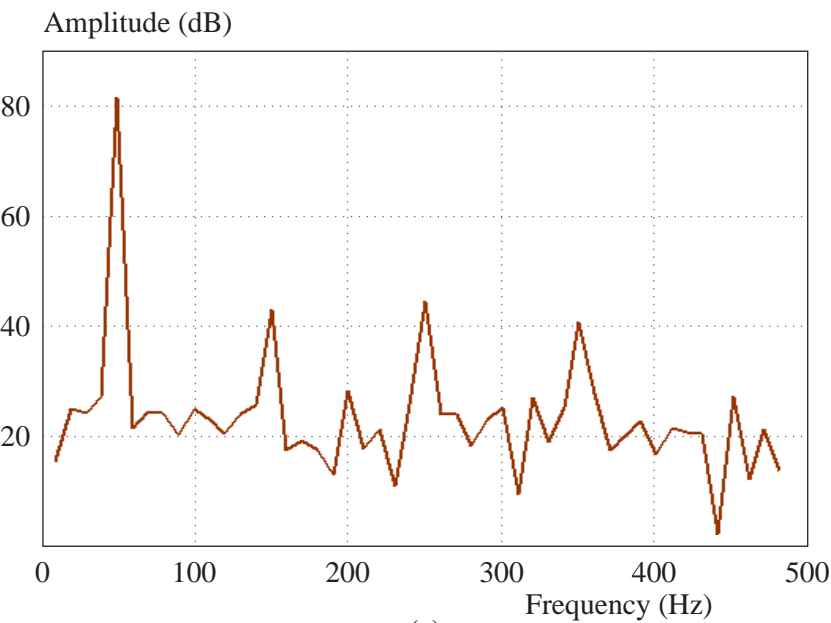

(a)

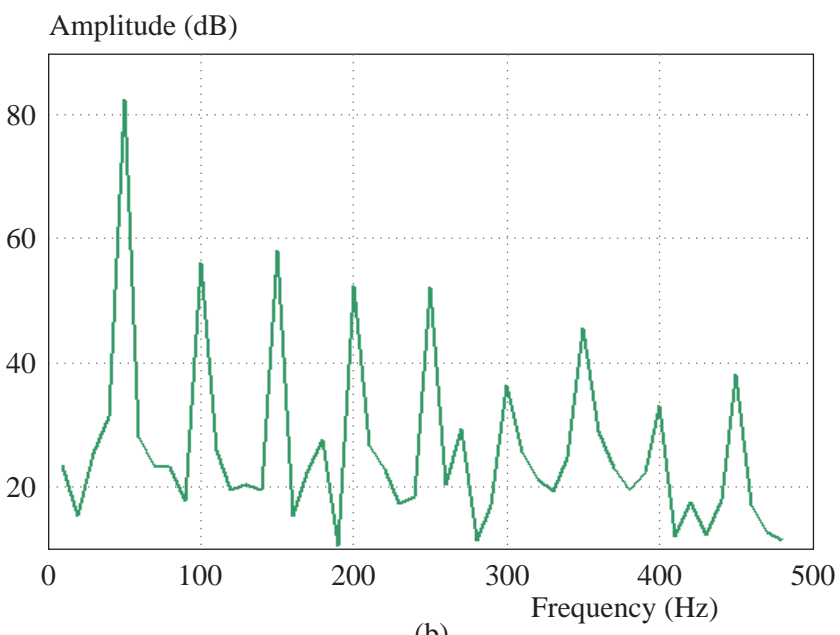

(b)

Fig. 11. The frequency spectrum of current $i_{3}$ for DC currents $I_{0}=0$ (left)and for $I_{0}=0.5 \mathrm{~A}($ right)

magnetic storm prediction and in the subsequent intervention of the operators of the transmission system. At work [12], various measures are proposed to protect transformers from the effects of magnetic storms.

\section{Acknowledgements}

Milan Stork's participation was supported by Department of Applied Electronics and Telecommunications, University of West Bohemia, Plzen, Czech Republic and by the Ministry of Education, Youth and Sports of the Czech Republic under the RICE New Technologies and Concepts for Smart Industrial Systems, project No. LO1607 and the Internal Grant Agency of University of West Bohemia in Plzen, the project SGS-2018-001

\section{REFERENCES}

[1] E. Priest, Solar magnetohydrodynamics, Dordecht, D. Reidel Publ.Co., 1984.

[2] D. Mayer, "Contribution to the investigation of the influence of geomagnetic storms on the electrification system", Acta Technica $A V C R$, vol. 58, no. 4, pp. 351-365, 2013.

[3] R. Girgis, "Effects of geomagnetically induced currents on power transformers and power systems", CIGRE, 21, rue d'Artois, F-75008 Paris, A2-304, pp. 1-8.

[4] P. R. Price, "Geomagnetically induced current effects on transformers", IEEE Trans. on Power Delivery, vol.17, no.4, 2002.

[5] K. J. Patel, "An analytic review of geomagnetically induced current effects in power system", Internat. Conf. on Electrical, Electronics and Optimization Techniques (ICEEOT), Issue Data 3-5 March 2016.

[6] D. Botler, "Methodology for simulation of geomagnetically induced currents in power systems", Space Wheather Space Clim., 4 A21, pp.2-11, 2014.

[7] A. Rezaei-Zare, "Analysis of three-phase transformer response due to GIC using an advanced duality-based model", IEEE Trans. on Power Delivery, vol. 31, no. 5, pp.2342-2350, 2015.

[8] F. Bachinger et al, "Direct current in transformers: effects and compensation", CIGRE Session 2012, Paris, France, August 27-31, SC-A2.

[9] H. Weng, "The impact of GIC on system voltage and generator output", 5-th Internal Conf. on Electrical, Utility, Deregulation and Restructuring of Power Technologies (DRPT), 26-29 2015.
[10] J. Ramírez-Niňo, "Core saturation effects of geomagnetic induced currents in power transformers", Journ. of Appl. Res. And Technology, 14, pp. 87-92, 2016.

[11] N. Nishiura, S. Yamashita and S. Kano, "Simulation analysis of geomagnetically-induced currents (GIC) effects on shell-form transformers", Power and Energy Society General Meeting (PES), IEEE, issue Data 21-25 July 2013.

[12] A. Rezaei-Zare, "Reactive power loss versus GIC characteristic of single-phase transformers", IEEE Trans. on Power Delivery, vol. 31, no. 5, pp. 2342-2350, 2015.

13] V. Gurevich, "Protection of power transformers against geomagnetically induced currents", Serbian Journ. of El. Eng., vol. 8, no. 2, pp. 333-339, 2011.

[14] J. R. Niňo, "Core saturation effect of geomagnetic induced currents in power transformers", Journal. of Applied Research and Technology, 14 (2016), pp.87-92, 2011.

[15] S. Lu, "Harmonics generated from DC based transformers", IEEE Trans. Power Delivery, vol.8, pp.725-731, 1993.

\section{Received 1 November 2018}

Milan Stork received the MSc degree in Electrical Engineering from the Technical University of Plzen, Czech Republic at the Department of Electronics in 1974 and $\mathrm{PhD}$ degree in automatic control systems at the Czech Technical University in Prague in 1985. In 1997, he became an Associate Professor at the Department of Applied Electronics and Telecommunication, faculty of Electrical Engineering on University of West Bohemia in Plzen, Czech Republic and full professor in 2007. He has numerous journal and conference publications. He is member of editorial board magazine "Physician and Technology". His research interests include analog/digital systems, signal processing and biomedical engineering, especially cardiopulmonary systems.

Daniel Mayer (Prof, Ing, DrSc) received the Ing, $\mathrm{PhD}$ and DrSc degrees in electrical engineering from Technical University in Prague, Czech Republic. In 1959 Associate Professor at the University of West Bohemia in Pilsen, in 1968 full Professor of the Theory of Electrical Engineering. Many years he was head of the Department of Theory of Electrical Engineering. Research interests: circuit theory, electromagnetic ?eld theory, electrical machines and apparatus, history of electrical engineering. He published 7 books, more than 340 scienti?c papers and 11 patents. He is a member of editorial boards of several international journals and leader of many grant projects. 